\title{
Variable Speed of Light Theories
}

\author{
J. W. Moffat \\ Department of Physics, University of Toronto, Toronto, Ontario, Canada \\ and \\ Perimeter Institute for Theoretical Physics, Waterloo, Ontario, Canada
}

\begin{abstract}
Two variable speed of light models and their physical consequences are investigated.
\end{abstract}

Talk given at the workshop on varying fundamental constants, JENAM 2002, Porto, Portugal, September 2-7, 2002. To be published in the proceedings by Kluwer publications.

\section{Varying Speed of Light Cosmology: An Alternative to In- flation}

It is ten years ago that an alternative solution to the initial value problems of cosmology based on a variable speed of light (VSL) was published [1]. The model was based on the idea that in the very early universe at a time $t \sim t_{P} \sim 10^{-43}$ sec., where $t_{P}$ denotes the Planck time, the local Lorentz invariance of the ground state of the universe was spontaneously broken by means of a non-zero vev of a vector field, $\left\langle\phi^{a}\right\rangle_{0} \neq 0$, where $a$ labels the flat tangent space coordinates of four-dimensional spacetime. At a temperature $T<T_{c}$, the local Lorentz symmetry of the vacuum was restored corresponding to a "non-restoration" of the symmetry group $S O(3,1)$ as the temperature $T$ increase 1

Does there exist an alternative to inflation, which can successfully allow a quantum field theory calculation of a scale invariant primordial spectrum?

\footnotetext{
${ }^{1}$ Models of such a non-restoration of symmetry in which a larger symmetry group breaks down to a smaller one as the temperature increases and passes through a critical temperature $T_{c}$ exist in the literature, see [2] for references.
} 
In spite of the successes of inflation theory, it is important to seek alternatives to it to see whether a different scenario could overcome some of the shortcomings of inflation, such as the problem of vacuum energy, the fine-tuning of the coupling constant to give the correct density profile in the present universe, and the unnaturally flat potentials needed to solve the initial value problems.

In the following, we shall consider anew the variable speed of light cosmology associated with a spontaneous symmetry breaking of Lorentz invariance, and a phase transition in the speed of light in the very early universe. A vierbein $e_{\mu}{ }^{a}$ is used to convert $\phi^{a}$ in flat tangent space into a 4-vector in coordinate space: $\phi^{\mu}=e_{a}{ }^{\mu} \phi^{a}$. We introduce a variable speed of light with $c(x)=\bar{c} \chi(x)$, where $\bar{c}$ is a constant with dimensions of velocity and $\chi(x)$ is a scalar field. The total action of the theory is $S=S_{G}+S_{M}+S_{\phi}+S_{\chi}$, where

$$
S_{G}=-\frac{c^{4}}{16 \pi G} \int d^{4} x e(R+2 \Lambda),
$$

$S_{M}$ is the matter action and

$$
S_{\phi}=\int d^{4} x \sqrt{-g}\left[\frac{1}{2} D_{\mu} \phi_{a} D^{\mu} \phi^{a}-V(\phi)\right] .
$$

Moreover, the action $S_{\chi}$ is

$$
S_{\chi}=\int d^{4} x \sqrt{-g}\left[\frac{1}{2} D_{\mu} \chi D^{\mu} \chi-V(\chi)-V(\chi \phi)\right],
$$

and $D_{\mu}$ is the covariant derivative operator: $D_{\mu}=\partial_{\mu} \delta_{b}^{a}+\left(\Omega_{\mu}\right)_{b}^{a}$ where $\left(\Omega_{\mu}\right)_{b}^{a}$ is the spin, gauge connection. 2

If the "Mexican hat" potential $V$ has a minimum at $\phi_{a}=v_{a}$, then the spontaneously broken solution is given by $v_{a}^{2}=\mu / 4 \lambda$. We can choose $\phi_{a}$ to be $\phi_{a}=\delta_{a 0} v=\delta_{a 0}\left(\mu^{2} / 4 \lambda\right)^{1 / 2}$. All the other solutions of $\phi_{a}$ are related to this one by a Lorentz transformation. Then, the homogeneous Lorentz group $S O(3,1)$ is broken down to the spatial rotation group $O(3)$. The three rotation generators $J_{i}(i=1,2,3)$ leave the vacuum invariant, $J_{i} v_{i}=0$, while the three Lorentz-boost generators $K_{i}$ break the vacuum symmetry,

\footnotetext{
${ }^{2}$ We demand that $\phi^{a}\left(\phi^{\mu}\right)$ be a timelike vector which ensures that the kinetic energy term $D_{\mu} \phi_{a} D^{\mu} \phi^{a}>0$ for all events in the past and future light cones of the flat tangent space, which avoids the occurrence of negative energy modes in the Hamiltonian. We could add a Lagrange multiplier term to the action to guarantee the timelike nature of the vector $\phi^{a}$. An alternative kinetic energy term would be $\left(\nabla_{\mu} \phi^{\mu}\right)^{2}$, which has no negative energy states (private communication: M. A. Clayton).
} 
$K_{i} v_{i} \neq 0$. In the spontaneously broken Lorentz symmetry phase, we can now have the speed of light $c$ undergo a phase transition, since we are no longer required to satisfy Einstein's second postulate of special relativity, namely, that the speed of light is a constant with respect to all local inertial frame observers.

Before the phase transition at a time $t \sim t_{c}$, the radiation density and the entropy of the universe, which are proportional to $c^{-3}$, are reduced by many orders of magnitude for $c \gg c_{m}$ (where $c_{m}=299792458 \mathrm{~m} \mathrm{~s}^{-1}$ is the currently measured speed of light), allowing for a semiclassical quantum field theory calculation of a scale invariant fluctuation spectrum. After the phase transition has occurred, the radiation density and the entropy of the universe increase hugely as $c \rightarrow c_{m}$, and the increase in entropy follows the arrow of time determined by the spontaneously broken direction of the vev $\left\langle\phi^{a}\right\rangle_{0}$. This solves the enigma of the arrow of time and the second law of thermodynamics.

The phase transition in the speed of light with $\dot{c} / c_{m}<0$ and $\log _{10}\left(c / c_{m}\right) \geq$ 30 solves the horizon and flatness problems with positive radiation pressure and density.

We shall consider a simple model of a free, minimally coupled scalar field $\psi$, which we identify with our physical field $\psi$ in the "unitary gauge' after the three Goldstone modes have been removed in our model of spontaneous symmetry breaking of Lorentz invariance. We choose for simplicity flat spacetime with $k=0$. The scalar field $\psi$ is pictured as a plane wave mode with coordinate wave vector $\vec{k}: \psi(\vec{x}, t)=\psi_{k}(t) \exp (i \vec{k} \cdot \vec{x})$, which satisfies

$$
\ddot{\psi}_{k}+3 H \dot{\psi}_{k}+\frac{c^{2} k^{2}}{R^{2}} \psi_{k}=0
$$

where have defined $\psi_{k}=\frac{1}{(2 \pi)^{3 / 2}} \int d^{3} x \psi(\vec{x}) \exp (-i \vec{k} \cdot \vec{x})$.

We consider that the quantum fluctuation modes are created in the spontaneously broken ground state and that their proper wavelength $\lambda_{p}=R / k$ is tiny compared to the Hubble radius, $R_{H}=c / H$ [2]. Moreover, a possible solution of the equations of motion for $\chi$ obtained from the action (3) is $c(t)=a / t^{b}+c_{0} \theta\left(t_{c}-t\right)+c_{m} \theta\left(t-t_{c}\right)$. Our equation of motion for $\psi_{k}$ is of the same form as the harmonic oscillator equation with a unit mass, a variable spring constant $c^{2} k^{2} / R^{2}$, and a variable friction damping coefficient $3 H$. For $\dot{\psi}_{k} \sim H \psi_{k}$, a radiation dominated universe $\left(p=\frac{1}{3} \rho\right.$ and $\left.H \sim 1 / t\right)$ and $t \rightarrow 0$ the proper wavelength, $\lambda_{p}$, of a mode is much smaller than the Hubble radius $R_{H}$, and the mode oscillates like an ordinary harmonic oscillator with small damping. However, when $t$ increases $\lambda_{p}$ will eventually 
become greater than $R_{H}$, and the mode enters an overdamped phase with $\dot{\psi}_{k} \sim 0$ and the mode "freezes". The ground state of the oscillator at some fixed time $t$ has the form of a Gaussian wave function with a spread given by $\left(\Delta \psi_{k}\right)^{2}=1 /\left(2 R^{2} c k\right)$.

For proper wavelengths much smaller than the Hubble radius, the universe evolves adiabatically, whereas for wavelengths larger than the Hubble radius, the overdamped modes cease to oscillate and $\Delta \psi_{k}$ will become constant. After the comoving wavelengths pass through the horizon they freeze and the spectrum spread is given by $\left(\Delta \psi_{k}\right)_{h}^{2}=1 /\left(2 R_{h}^{2} c_{h} k\right)$, where $c_{h}$ and $R_{h}$ are the values of $c$ and $R$ at the time the modes cross the Hubble radius, $R_{H}$, i.e. when $R_{h} / k=c_{h} / H_{h}$.

The fluctuation modes at later times have the spectrum $\left(\Delta \psi_{k}\right)_{h}^{2} \sim$ $H_{h}^{2} / c_{h}^{3} k^{3}$. This constitutes the prediction of a scale invariant spectrum with $k^{3}\left|\delta_{k}\right| \sim$ constant, where $\delta_{k}$ is the fractional energy density fluctuation in

momentum space. We observe that at later times: $\left(R / R_{h}\right)^{2}\left(c_{0} / c_{h}\right)\left(\Delta \psi_{k}\right)^{2}=$ $\left(\Delta \psi_{k}\right)_{h}^{2}$. We see that for $c_{h}=c_{m}$, the spread $\left(\Delta \psi_{k}\right)^{2}$ is magnified by the huge factor $\left(c / c_{m}\right) \sim 10^{30}$, so that the late time quantum fluctuations have macroscopically relevant cosmological interest. In inflation theory, it is the factor $\left(R / R_{h}\right)^{2}$ that is exponentially enhanced and also produces macroscopically large fluctuation effects.

We have succeeded in obtaining a viable VSL model, which predicts a scale invariant, Gaussian and adiabatic spectrum that agrees with the prediction of inflation and the observational data. The semi-classical approximation used in our quantum field theory calculation is justified, for the radiation energy density and the entropy are severely diluted due to the large value of $c$ before the phase transition. A fundamental difference between VSL cosmology and inflation is that we can choose the cosmological constant $\Lambda$ to be small or zero from the beginning of the universe.

\section{Bimetric Gravity Theory, Dimming of Supernovae and Dark Energy}

In the bimetric scalar-tensor gravitational theory there are two frames associated with the two metrics $\hat{g}_{\mu \nu}$ and $g_{\mu \nu}$, which are linked by the gradients of a scalar field: $\hat{g}_{\mu \nu}=g_{\mu \nu}+B \partial_{\mu} \phi \partial_{\nu} \phi$ 酒.

The choice of a comoving frame for the metric $\hat{g}_{\mu \nu}$ or $g_{\mu \nu}$ has fundamental physical consequences for local observers in either metric spacetimes, while 
maintaining diffeomorphism invariance. When the metric $g_{\mu \nu}$ is chosen to be associated with comoving coordinates, then the speed of light varies in the frame with the metric $\hat{g}_{\mu \nu}$. We call this the variable speed of light (VSL) metric frame.

If we choose $\hat{g}_{\mu \nu}$ to be associated with comoving coordinates, then the speed of light is constant but the speed of gravitational waves varies with time (VSGW frame). The Friedmann equation in the VSGW frame is given by

$$
H^{2}+\frac{c_{m}^{2} k K}{R^{2}}=\frac{8 \pi G}{3} K^{3 / 2} \rho+\frac{1}{3} c_{m}^{2} \Lambda K+\frac{1}{6} \tilde{\rho}_{\phi},
$$

where we have defined: $\tilde{\rho}_{\phi}=\frac{1}{2} \dot{\phi}^{2}+c_{m}^{2} K V(\phi)$. We see that when $v_{g} \equiv$ $c_{m} K^{1 / 2}=c_{m}\left[1-\left(B / c_{m}^{2}\right) \dot{\phi}^{2}\right]^{1 / 2} \rightarrow 0\left(v_{g}\right.$ is the speed of gravitational wave propagation) in the early universe, then $H \rightarrow \dot{\phi} / \sqrt{2}$ and for $\dot{\phi}$ slowly varying, this produces an inflationary solution in the initial universe, without a "slow roll" potential approximation as in standard inflationary theories.

Observers in the VSL metric frame see the dimming of supernovae, because of the increase of the luminosity distance versus red shift, due to an increasing speed of light in the past universe, $\sim 10 \%$ between $z=0$ and $z \sim 2-3$. Moreover, in this frame the scalar field $\phi$ describes a dark energy component in the Friedmann equation for the cosmic scale without acceleration [5]. On the other hand, an observer in the VSGW metric frame will observe the universe to be accelerating and the supernovae will appear to be farther away.

Our bimetric gravity theory predicts that the gravitational constant $G$ can vary in spacetime, while the fine-structure constant, $\alpha=e^{2} / \hbar c_{m}$, does not vary with time, which allows for a $10-15 \%$ increase in the speed of light between $z=0$ and $z \sim 2-3$. The data of Webb et al. [6], showing a variation in $\alpha$ between $z=0.1$ and $z=3.5$, is still to be confirmed by independent observational data.

\section{References}

[1] J. W. Moffat, Int. J. Mod. Phys. D2, 351 (1993), arXive:gr-qc/9211020; J. W. Moffat, Found. of Phys. 23, 411 (1993), arXive:gr-qc/9209001; J. W. Moffat, Fluctuating Paths and Fields, Festschrift dedicated to Hagen Kleinert, edited by H. Jancke, A. Pelster, H.-J. Schmidt, and M. Bachmann, World Scientific, Singapore, p. 741, 2001, arXive:astro$\mathrm{ph} / 9811390$. 
[2] J. W. Moffat, arXive:hep-th/0208122.

[3] A. Albrecht and J. Magueijo, Phys. Rev. D59, 043516, (1999), arXive:astro-ph/9811018; J. D. Barrow, Phys. Rev. D59, 043515 (1999); J. D. Barrow and J. Magueijo, Phys. Lett. B477, 246 (1999), arXive:astro-ph/9811073 v2; R. H. Brandenberger and J. Magueijo, "Imaginative Cosmology", Invited Lectures given at the IPM School on Cosmology 1999: Large Scale Structure Formation, to be published by Kluwer, Dordrecht, arXive:hep-ph/9912247; J. Magueijo, Phys. Rev. D63, 043502 (2001), arXive:astro-ph/0010591.

[4] M. A. Clayton and J. W. Moffat, Phys. Lett. B460, 263 (1999), arXive:astro-ph/9812481; M. A. Clayton and J. W. Moffat, Phys. Lett. B477, 269 (2000), arXive:gr-qc/9910112; M. A. Clayton and J. W. Moffat, Int. J. of Mod. Phys. D11, 187 (2002), arXive:gr-qc/0003070; M. A. Clayton and J. W. Moffat, Phys. Lett. B506, 177 (2001), arXive:gr-qc/0101126 v2; I. T. Drummond, Phys. Rev. D63 043503 (2001), arXive:astro-ph/0008234; M. A. Clayton and J. W. Moffat, arXive:astro-ph/0203164; B. A. Bassett, S. Liberati, C. Molinari-Paris, and M. Visser, Phys. Rev. D62 103518 (2000), arXive:astro-ph/0001441 v2; D. Youm, Phys. Rev. D63 (2001) 125011; arXive:hep-th/0102194;

Phys. Rev. D65 025008 (2002), arXive:hep-th/0108073; arXive:hepth/0108237.

[5] J. W. Moffat, to be published in Int. J. Mod. Phys. (2002), arXive:grqc/0202012.

[6] J. K. Webb et al., Phys. Rev. Lett. 82, 884 (1999), arXive:astro$\mathrm{ph} / 9803165$. 